\title{
Network Analysis Accelerates Understanding of Disease Mechanisms
}

\author{
Jose A Santiago and Judith A Potashkin*
}

Department of Cellular and Molecular Pharmacology, Rosalind Franklin University of Medicine and Science, USA

During the last decade, high throughput methods including gene profiling and genome wide association studies have identified thousands of genetic risk factors, biological pathways and biomarkers for a wide range of diseases. Despite this apparent success, the translation of this valuable data into clinical tools for disease diagnosis, prognosis and treatment remains challenging due to the rigorous and highly complex statistical methods used and the limited functional and biological information derived from these datasets. To address this hurdle, network biology has emerged as a powerful tool for the characterization of complex diseases by integrating genetic and environmental factors into a single system with biological relevance.

Based on the observation that causal genes of disease tend to be interconnected in common biological modules [1], the study of how disruption in these genetic networks lead to disease has been fundamental in the understanding of many complex diseases. In this regard, network biology has elucidated molecular networks causative of disease. For example, integration of co-expression networks and genotypic data identified networks associated with metabolic disease and causal genes for obesity $[2,3]$. An alternative method to discover novel causative disease genes is to explore the interconnectivity and distance of well-characterized genes to closely associated neighbors in a gene or protein interaction network. Using this strategy, novel susceptibility genes and underlying disease mechanisms were identified in Alzheimer's disease (AD) [4].

Another area of great interest is understanding disease comorbities. Network approaches have been useful in dissecting and providing insight into the underlying mechanism leading to concurrent diseases. Remarkably, analysis of the human metabolic network revealed that connected diseases with metabolic links displayed higher comorbidity than those with no metabolic links [5]. Given the intriguing hypothesis of a shared mechanisms leading to Parkinson's disease and diabetes [6], this approach could provide insights into this relationship.

Biomarker discovery is another area of promise in the field of network biology. There are several challenges in the discovery and validation of biomarkers from high-throughput studies. One problem arising is that biomarkers from microarray studies can be data setspecific and provide limited information about the underlying disease etiology. Also, the biomarker signature identified depends on the statistical approach used in the analysis. In addition, it has become evident that there is very little overlap between biomarker sets identified for a particular disease using similar gene profiling methods. To address these issues, network based approaches are expected to facilitate the integration of multiple lines of information to identify accurate and reliable biomarkers for disease diagnosis. In this context, network approaches have successfully identified biomarkers with clinical applicability. For example, using a well characterized set of genes, a network approach identified biomarkers for progressive supranuclear palsy [7]. This approach utilizes a random walk algorithm with restart in which a random walker moves from a known disease gene to a random neighbor within a specified distance in the functional linkage network [8]. Biosignatures for colorectal cancer were also identified using this approach [9].

In summary, network-based approaches provide an innovative framework to dissect complex diseases. With the emerging field of RNA-sequencing technology, the amount of data and information is expected to greatly increase; therefore, integrative network approaches will be valuable to dissect the relevant biological information. Based on the recent success in identifying biomarkers for disease diagnosis, we foresee that network-based approaches will facilitate the discovery of therapeutic targets. Further, we expect that networks and systems biology will accelerate the translation of biomarkers and therapeutics to the clinic.

\section{References}

1. Oti M, Brunner HG (2007) The modular nature of genetic diseases. Clin Genet 71: 1-11.

2. Chen Y, Zhu J, Lum PY, Yang X, Pinto S, et al. (2008) Variations in DNA elucidate molecular networks that cause disease. Nature 452: 429-435.

3. Yang X, Deignan JL, Qi H, Zhu J, Qian S, et al. (2009) Validation of candidate causal genes for obesity that affect shared metabolic pathways and networks. Nat Genet 41: 415-423.

4. Soler-López M, Zanzoni A, Lluís R, Stelzl U, Aloy P (2011) Interactome mapping suggests new mechanistic details underlying Alzheimer's disease. Genome Res 21: 364-376.

5. Lee DS, Park J, Kay KA, Christakis NA, Oltvai ZN, et al. (2008) The implications of human metabolic network topology for disease comorbidity. Proc Natl Acad Sci U S A 105: 9880-9885.

6. Santiago JA, Potashkin JA (2013) Shared dysregulated pathways lead to Parkinson's disease and diabetes. Trends Mol Med 19: 176-186.

7. Santiago JA, Potashkin JA (in press) A Network Approach to Diagnostic Biomarkers in Progressive Supranuclear Palsy. Mov Disord.

8. Köhler S, Bauer S, Horn D, Robinson PN (2008) Walking the interactome for prioritization of candidate disease genes. Am J Hum Genet 82: 949-958.

9. Shi M, Beauchamp RD, Zhang B (2012) A network-based gene expression signature informs prognosis and treatment for colorectal cancer patients. PLoS

*Corresponding author: Judith A Potashkin, Department of Cellular and Molecular Pharmacology, The Chicago Medical School, Rosalind Franklin University of Medicine and Science, 3333 Green Bay Rd, North Chicago, Illinois 60064-3037, Tel: (847) 5788677; Fax: (847) 578-3268; E-mail: judy.potashkin@rosalindfranklin.edu

Received October 16, 2013; Accepted October 17, 2013; Published October 25 2013

Citation: Santiago JA, Potashkin JA (2013) Network Analysis Accelerates Understanding of Disease Mechanisms. Clin Exp Pharmacol 3: e123. doi:10.4172/2161-1459.1000e123

Copyright: (c) 2013 Santiago JA, et al. This is an open-access article distributed under the terms of the Creative Commons Attribution License, which permits unrestricted use, distribution, and reproduction in any medium, provided the original author and source are credited. 